\title{
Coulomb focusing effect on the space distribution of the rescattering electron wavepacket in the laser-atom interaction
}

\author{
K. Sasaki ${ }^{1}$, X. M. Tong ${ }^{1,2}$, and N. Toshima ${ }^{1}$ \\ ${ }^{1}$ Institute of Materials Science, Graduate School of Pure and Applied Sciences, \\ University of Tsukuba, 1-1-1 Tennodai, Tsukuba, Ibaraki 305-8573, Japan \\ ${ }^{2}$ Center for Computational Sciences, University of Tsukuba, 1-1-1 Tennodai, \\ Tsukuba, Ibaraki 305-8577, Japan \\ E-mail: tong.xiaomin.ga@u.tsukuba.ac.jp
}

\begin{abstract}
We investigated the Coulomb focusing effect on the space distribution of the rescattering electron wavepacket created in the laser-atom interaction solving the time integral equation. The Coulomb focusing is conspicuous in the even-number returns since the rescattering electron energies are lower there. The Coulomb force focuses the rescattering electron beam into a small space and the rescattering electron beam current intensity can reach the order of $10^{10} \mathrm{~A} / \mathrm{cm}^{2}$, much more intense than the conventional electron beam used in scattering experiments. The Coulomb focusing effect plays a less important role for the first return and this explains why the released kinetic energy spectra have a peak at the third return in the experiments of the double ionization of $\mathrm{H}_{2}$ molecules.
\end{abstract}

PACS numbers: $32,80 . \mathrm{Fb}, 32.80 . \mathrm{Wr}, 42.65 . \mathrm{Re}$

Submitted to: J. Phys. B: At. Mol. Opt. Phys.

The laser-atom interaction is a hot research topic stimulated by the rapid advance of the laser technology. Most of the observed phenomena can be explained by the rescattering model proposed by Corkum [1] as shown in Fig. 1. The electron is firstly released by the laser through the tunneling ionization process when the laser field reaches its peak strength and the released electron is bounced back when the laser field changes the polarization direction. The collision of the bounced electron with the parent core is similar to the electron beam scattering with the target parent core. In principle, all the dynamics which can be investigated by the conventional electron beam scattering can be also studied by the rescattering electron beam. More attractively, the rescattering electron beam is a coherent beam and the beam intensity is much higher than the conventional one. Thus, the application of the rescattering electron beam provides a potential way to study molecular structure imaging [2], like the conventional (e, 2e) experiment [3]. For the conventional electron beam experiment, the beam intensity, the electron energy and other physical conditions can be controlled 


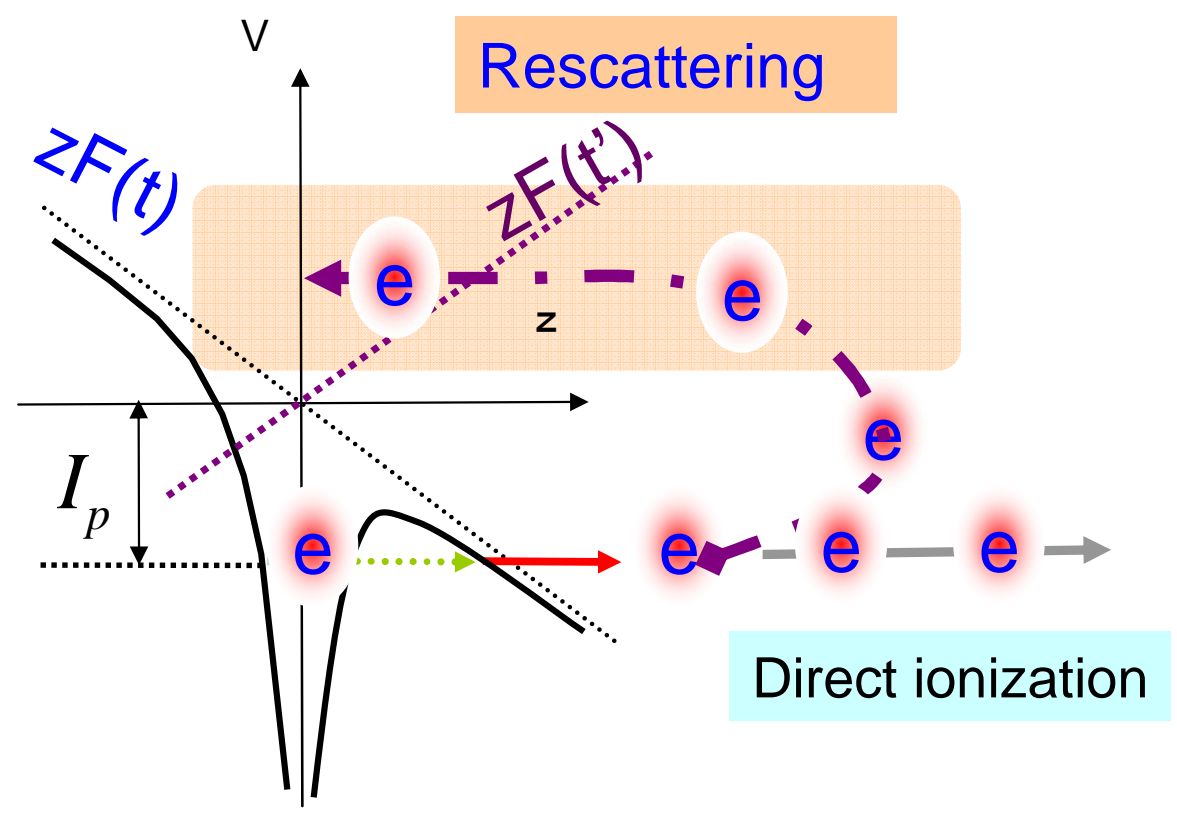

Figure 1. (Color online) The schematic of the rescattering process in the laser-atom interactions.

directly. For the rescattering electron beam case, the detailed information [4], such as the energy distribution and the space distribution, is not known. Without this knowledge, the beam quality cannot be controlled experimentally. Since the rescattering is an intermediate process as shown in Fig. 1, the information cannot be obtained directly from the experimental measurement. We have to rely on the numerical simulation.

The dynamical information can be obtained by solving the time-dependent Schrödinger equation numerically. However, getting the rescattering information directly from numerical calculations is not easy because (1) the wavefunction of the ejected electron is only a small fractional part of the total wavefunction; (2) the rescattering electron wavepackets created at different laser cycles mix up making it difficult to decompose the $n$th returns separately. Of course, the rescattering information can be also obtained by a semiclassical simulation [5] but the reliability of the classical results is not clear since the phase information is missing. In our previous work [6], we proposed a numerical procedure to obtain the rescattering information directly by solving the time-integral equation instead of the differential Schrödinger equation and we succeeded in extracting the scattering details. Morishita et al. [7] proposed an alternative way to extract the rescattering information from the above threshold ionization. Xie et al. [8] also obtained the rescattering momentum distribution in the first return by directly solving the time-dependent Schrödinger equation. So far most of the works focused on the returning time and the energy (or momentum) distribution and, to the best of our knowledge, there is no report on the space distribution of the scattering electron, which is another crucial information to image the molecular structures using rescattering electron beam. The purpose of the present paper is to study the space distribution of the rescattering electron beam intensity in a full quantum, 
nonperturbative method.

We transform the time-dependent Schrödinger equation into an integral equation [6] and factor out the unperturbed part corresponding to the initial state propagating without interacting with the laser field. The time-dependent wavefunction is written as (atomic units $\hbar=m=e=1$ are used)

$$
\Psi(t)=-i \int_{t_{0}}^{t_{1}} e^{-i \int_{\tau}^{t}\left[H_{0}+V\left(t^{\prime}\right)\right] d t^{\prime}} V(\tau) e^{-i H_{0} \tau} \Phi_{0} d \tau .
$$

Here $\Phi_{0}$ is the initial state, $H_{0}$ is the laser-field free Hamiltonian (we take hydrogen atom as an example) and $V(t)=-z E_{0} \cos \omega t$ is the laser electron interaction. $\Psi(t)$ is interpreted as the time dependent wavefunction corresponding to the electron ionized by the laser field during the time interval from $t_{0}$ to $t_{1}$, with $E_{0}$ the laser field strength and $\omega$ the laser frequency. The laser intensity is $E_{0}^{2} / 2$. The time-propagation is performed by the split-operator method with a generalized pseudospectral grid in the energy representation $[9,10]$. The laser-atom interaction $V$ appears twice in the above equation. The intuitive physical picture is that the atom is ionized due to the laser-atom interaction $V(\tau)$ at time $\tau$ and the ejected electron is propagated in the atomic field combined with the laser field. Because the laser field oscillates periodically, we have only to trace the motion of the electron ejected by the laser field in a half cycle. Without loss of generality, we choose $\omega t_{0}=-\pi / 2$ and $\omega t_{1}=\pi / 2$ in the following discussion and follow the electron motion for $t \geq t_{1}$. When the ejected electron revisits the parent core, the time-dependent wavefunction $\Psi(t)$ in the inner-region can be rewritten as

$$
\Psi(\mathbf{r}, t)=\int C_{l}(E, t) \psi_{l}(E, r) Y_{l 0}(\hat{\mathbf{r}}) d E,
$$

with

$$
C_{l}(E, t)=\int f\left(r, R_{c}\right) Y_{l 0}^{*}(\hat{\mathbf{r}}) \psi_{l}^{*}(E, r) \Psi(t) d \mathbf{r} .
$$

Here $\psi_{l}^{*}(E, r)$ is the laser-field-free electron wavefunction for a given partial wave $l$ and energy $E>0$, and $Y_{l 0}^{*}(\hat{\mathbf{r}})$ is the spherical harmonic. $f\left(r, R_{c}\right)$ is introduced to define the size of the inner-region $R_{c}$ with the boundary width $\Delta$ as

$$
f\left(r, R_{c}\right)=\left\{\begin{array}{ll}
1.0 & \text { for } \quad r<R_{c} \\
e^{-\left(r-R_{c}\right)^{2} / \Delta^{2}} & \text { for } \quad r \geq R_{c}
\end{array} .\right.
$$

In the present calculation, $R_{c}$ is 5 to 10 a.u. and $\Delta$ is 2 a.u. The results are not sensitive to the choice of $R_{c}$ and $\Delta$. At time $t$ from the coefficients $\left\{C_{l}(E, t)\right\}$, we obtain the energy distribution of the rescattering electron wavepacket as

$$
\frac{d P(E, t)}{d E}=\sum_{l}\left|C_{l}(E, t)\right|^{2}
$$

Because the impact parameter $b$ is not well defined in the quantum mechanics, we approximate it as $b^{2}=l(l+1) /(2 E)$, which corresponds to the impact parameter when the electron is incident from infinite separation, and define the rescattering beam intensity as

$$
I(b, t)=\frac{d P(b, t)}{2 \pi b d b}=\int \sum_{l} \frac{\sqrt{2 E}}{\pi}\left|C_{l}(E, t)\right|^{2} \delta\left(b^{2}-\frac{l(l+1)}{2 E}\right) d E .
$$




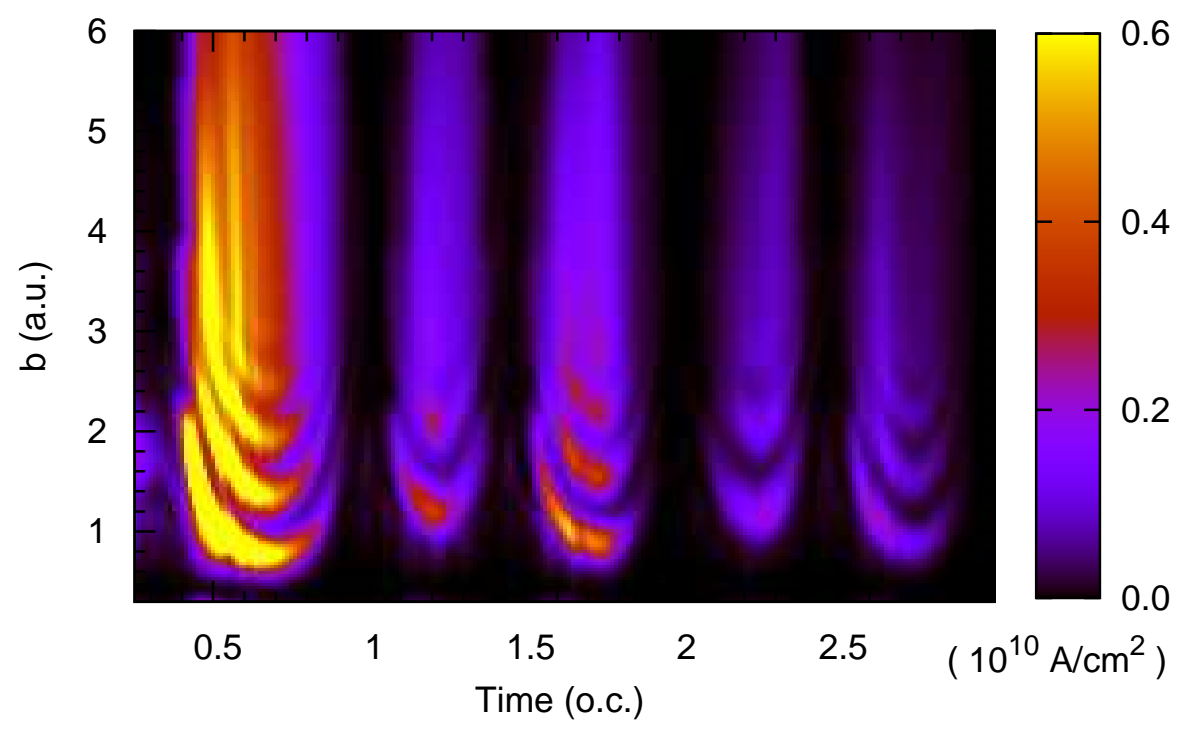

Figure 2. (Color online) The rescattering electron intensity as a function of the rescattering time and the impact parameter $b$ from the quantum calculation. The time is in the unit of laser optical cycle (o.c.).

Note that the electron velocity $v=\sqrt{2 E}$ is multiplied to the integrand. In the present simulation, we choose the laser peak intensity as $2 \times 10^{14} \mathrm{~W} / \mathrm{cm}^{2}$ and wavelength as $800 \mathrm{~nm}$ (the parameters most commonly used in the experiments). Figure 2 shows the rescattering electron beam intensity $I(b, t)$. To compare with the conventional electron beam intensity, we convert the rescattering electron beam intensity from atomic unit to $\mathrm{A} / \mathrm{cm}^{2}$. We see clearly that the rescattering electron revisits the parent core at the returning times predicted by the simple classical model [1] and the electron beam intensity reaches as high as $10^{10} \mathrm{~A} / \mathrm{cm}^{2}$, which is several orders of magnitude more intense than the electron beam intensity in the electron beam ion trap [11], which is of the order of $10^{3} \mathrm{~A} / \mathrm{cm}^{2}$. Note that the beam intensity obtained in the present work is about one order of magnitude smaller than the one predicted by the simple model [12], in which the size of the rescattering wavepackets was underestimated. The space distribution is not smooth but contains several strips within a give returning time. These strips come from the discretized angular momenta. The left end peak in Fig. 2 corresponds to the first return, then the next one to the second return and so on. We see the space distribution is rather smooth and uniform for the first return. In the third return, the electron is concentrated in the inner region, which corresponds to the close collision with the parent core. We attribute this character to the Coulomb focusing effect. To explain the Coulomb focusing effect, we show the time-dependent energy distribution of the rescattering electron in Fig. 3. For the first return, the rescattering energy takes the highest value which is higher than $3.17 U_{p}$ due to the Coulomb attraction as we discussed in our previous paper [6]. The highest first return energy decreases to $3.17 U_{p}$ as the laser intensity increases. This figure is similar to the ones plotted in Ref. [6] but the energy range is expanded in order to show the whole distribution. 


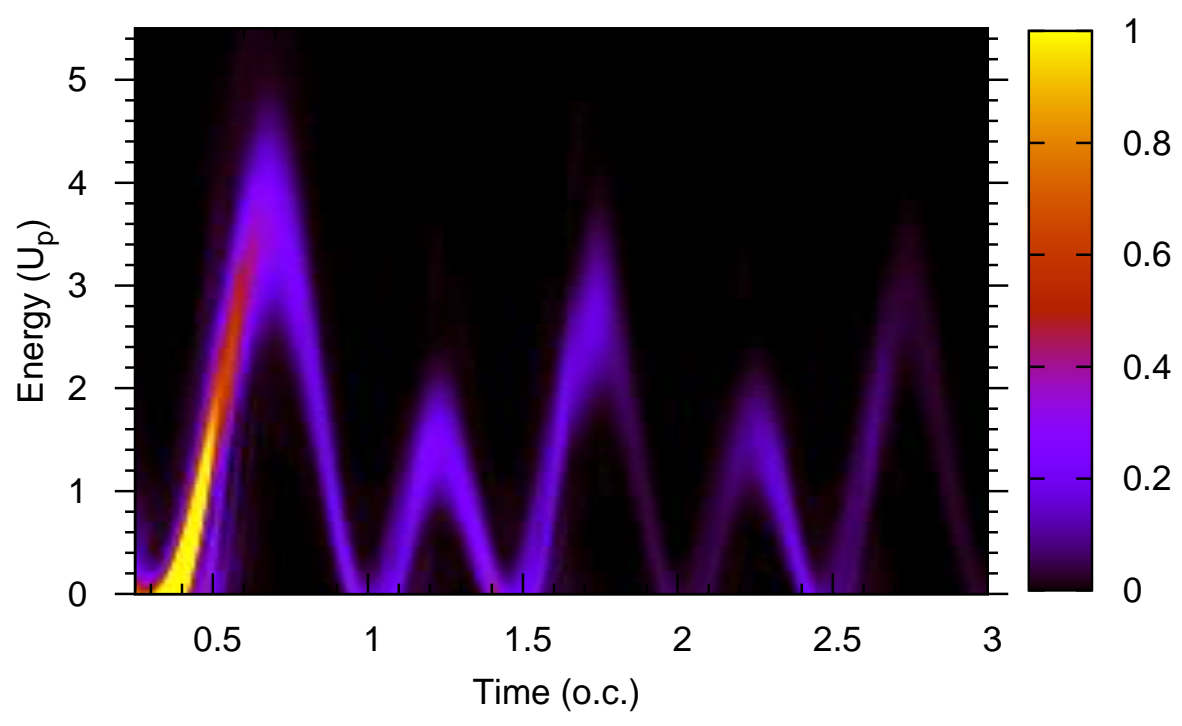

Figure 3. (Color online) The rescattering energy and time distributions. The color coding represents the relative value of $\frac{d P(E, t)}{d E}$.

Generally speaking, the rescattering energies of even returns are lower than those of odd returns. Among the odd returns, the rescattering energy of the first return is higher than the $3 \mathrm{rd}, 5$ th and so on. This is due to the fact that the high energy electron in the first return can not be bounced back by the laser field and is ionized after the first return. The Coulomb focusing effect is less important for the high energy electron since it spends shorter time in the inner region. When the electron returns to the parent core again (the second return), the electron energy is much lower than the first return energy and the returning electron beam is focused by the Coulomb interaction. The focused electron moves along the laser polarization direction in a narrow region on the perpendicular plane to the polarization direction. When the focused electron beam is bounced back by the laser field with a relatively high energy in the third return, the Coulomb force does not affect the motion of the returning electron significantly. But the electron has already been focused in a small region on the plane perpendicular to the polarization direction during the second return. Thus the Coulomb focusing effect in the second return results in a high intensity electron beam at the third return.

With the understanding of the details of the Coulomb focusing effect, we analyze the rescattering induced double ionization experiments $[12,13,14]$ for $\mathrm{H}_{2}$ molecules. The strong third return peak was explained in those papers to be due to that (a) the vibration wavepacket moving to the large nuclei separation $R$ results in a small ionization potential; (b) the third return energy is lower than the first return energy and closer to the ionization threshold or the peak position of the electron impact ionization cross section $[15,16]$. From the present study, we have seen that the Coulomb focusing effect at the second return also contribute to the enhancement of the kinetic energy release at the third return. In the previous semi-classical simulations $[15,16]$, such an effect was taken into account without analysis in detail. 


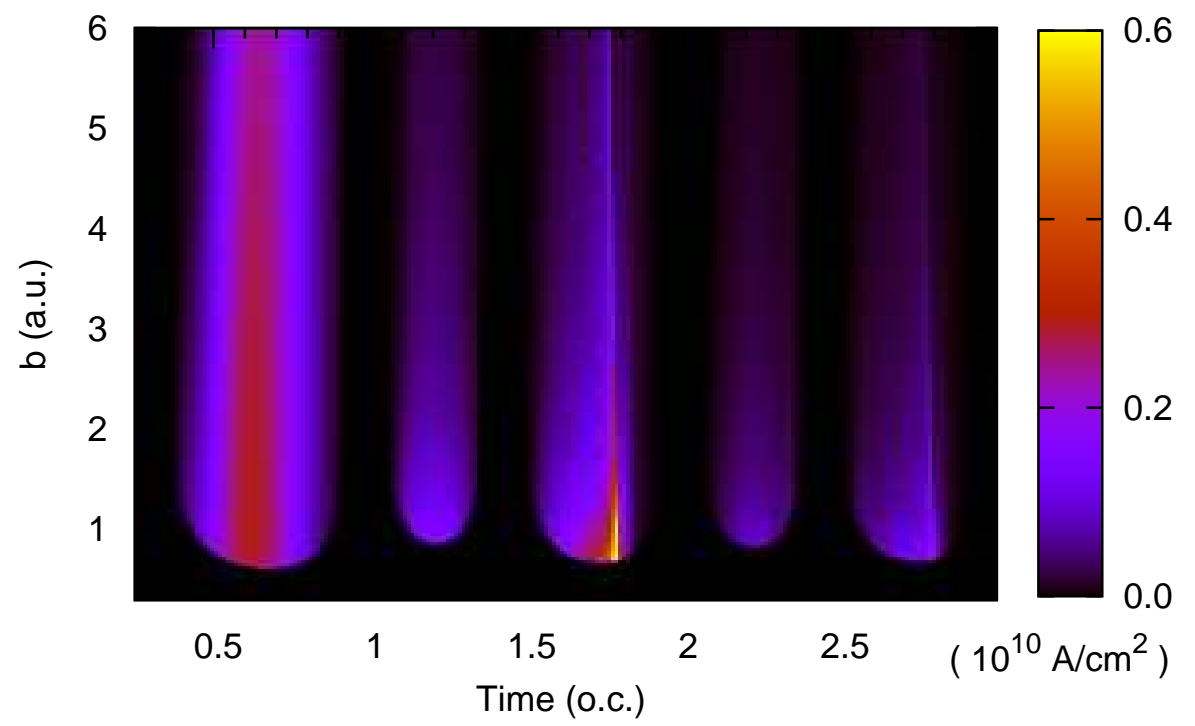

Figure 4. (Color online) The rescattering electron intensity as a function of the rescattering time and the impact parameter $b$ from the semi-classical simulation.

To cross check the reliability of the quantum calculations, we also calculated the space distribution of the rescattering electron by a semi-classical simulation [5]. In the simulation we monitor the impact parameter $b$-dependent rescattering electron beam intensity as shown in Fig. 4. The electron beam intensity distribution is rather smooth because of the nature of the classical behavior. The obtained results are consistent with the quantum calculations both in the returning times and the beam intensity. The Coulomb focusing effect on the third return is also clearly seen. The major physical conclusions, such as the smooth space distribution for the first return, are consistent in both calculations. There are still fundamental differences between the quantum simulation (Fig. 2) and the classical simulation (Fig. 4). For a given angular momentum, the high incident energy results in a small impact parameter and this explains why there are many curved strips in Fig. 2. The impact parameter decreases when the rescattering energy in Fig. 3 increases. The strips do not show up in the classical results because the angular momentum is not quantized.

To summarize, we have studied the rescattering electron space distribution when it revisits the parent core. The rescattering electron beam intensity can reach as high as $10^{10} \mathrm{~A} / \mathrm{cm}^{2}$, which is much higher than the conventional electron beams. We can do a similar simulation for molecules to obtain the rescattering electron energy and space distribution. Comparison with the measurement of the ejected electron distributions enables us to get the information on the electronic wavefunction analogously to the (e,2e) experiment. Thus, the understanding of the rescattering electron beam mechanism helps us to analyze the molecular electronic structure. 


\section{Acknowledgments}

This work was supported by Grant-in-Aids for Scientific Research (C) from the Japan Society for the Promotion of Science. The numerical calculations have been carried out by T2K-Tsukuba System in Center for Computational Sciences, University of Tsukuba.

\section{References}

[1] Corkum P B 1993 Phys. Rev. Lett. 71 1994-1997

[2] Itatani J, Levesque J, Zeidler D, Niikura H, Pepin H, Kieffer J C, Corkum P B and Villeneuve D M 2004 Nature $432867-871$

[3] Brion C E, Cooper G, Zheng Y, Litvinyuk I V and McCarthy I E 2001 Chem. Phys. 270 13-30

[4] Spanner M, Smirnova O, Corkum P B and Ivanov M Y 2004 J. Phys. B 37 L243

[5] Tong X M and Lin C D 2007 Phys. Rev. Lett. 98123002

[6] Tong X M, Watahiki S, Hino K and Toshima N 2007 Phys. Rev. Lett. 99093001

[7] Morishita T, Le A T, Chen Z and Lin C D 2008 Phys. Rev. Lett. 100013903

[8] Xie X H, Jordan G, Wickenhause M, Scrinzi A 2007 J. Mod. Opt. 54, 999-1010

[9] Tong X M and Chu S I 1997 Chem. Phys. 217 119-130

[10] Tong X M, Hino K and Toshima N 2006 Phys. Rev. A 74031405

[11] Nakamura N, Asada J, Currell F J, Fukami T, Hirayama T, Motohashi K, Nagata T, Nojikawa E, Ohtani S, Okazaki K, Sakurai M, Shiraishi H, Tsurubuchi S and Watanabe H Physica Scripta T73 362-364

[12] Niikura H, Legare F, Hasbani R, Bandrauk A D, Ivanov M Y, Villeneuve D M and Corkum P B 2002 Nature $417917-922$

[13] Alnaser A S, Osipov T, Benis E P, Wech A, Shan B, Cocke C L, Tong X M and Lin C D 2003 Phys. Rev. Lett. 91163002

[14] Alnaser A S, Tong X M, Osipov T, Voss S, Maharjan C M, Ranitovic P, Ulrich B, Shan B, Chang Z, Lin C D and Cocke C L 2004 Phys. Rev. Lett. 93183202

[15] Tong X M, Zhao Z X and Lin C D 2003 Phys. Rev. A 68043412

[16] Tong X M, Zhao Z X and Lin C D 2003 Phys. Rev. Lett. 91233203 